\title{
Resistance Exercise for Breast Cancer Patients? Evidence from the Last Decade
}

\author{
Lars Gerland Freerk T. Baumann Timo Niels \\ Department I of Internal Medicine, Center of Integrated Oncology, Aachen Bonn Cologne Düsseldorf, University \\ Hospital of Cologne, Cologne, Germany
}

\section{Keywords}

Resistance exercise · Breast cancer · Strength training ·

Muscle strength $\cdot$ Side effects

\begin{abstract}
Background: Breast cancer is associated with many therapyinduced side effects that impact patients' lives from diagnosis to long-term survivorship. Physical activity has become an important and proven supportive measure in treating side effects like loss of muscle strength, fatigue, chemotherapy-induced peripheral neuropathy, lymphedema, and loss of quality of life. Resistance training (RT) is an established exercise intervention for breast cancer patients, but the correct type, timing, intensity, and duration of exercise remain unclear. It is necessary to investigate different resistance training interventions and their effects on breast cancer patients by covering all stages of treatment, beginning with prehabilitation, through the period of acute therapy, to long-term survivorship. Conclusion: Upon evaluation of randomized controlled trials (RCTs) from the past decade, RT was found to be feasible and safe. Furthermore, there is evidence on the impact of RT on muscle strength, CRF and QoL amongst other factors. Studies implementing mixes of aerobic and strength exercises are rather common, but RCTs of RT-only protocols remain scarce. Different strength training protocols at distinct stages of breast cancer treatment have been conducted, but with the complexity of treatments and the variety of training styles, a large field of study remains. Key Messages: Although the overall data on RT for breast cancer patients has increased, there are many different methodological approaches and testing measures as well as
\end{abstract}

gaps in study documentation. There is still very little of the evidence that would facilitate the compilation of standardized and individualized guidelines.

๑) 2021 S. Karger AG, Basel

\section{Introduction}

Breast cancer is still the most common cancer diagnosis in women worldwide, and it is associated with many therapy-induced side effects which can remain a challenge for breast cancer patients for months, years, and even decades [1]. Exercise has been proven to benefit breast cancer patients in many ways, and it improves patients' physical condition and reduces treatment-associated impairments [2]. Both aerobic and resistance training (RT) are recommended as they involve different mechanisms. Even though RT is considered more promising in the domains of muscle mass, strength, and countering some side effects of therapy, the limited availability and lack of clarity of reported data mean that the ideal modalities, timing, intensity, and modes of RT have not yet been established.

The purpose of this review was to evaluate the findings of randomized-controlled trials (RCTs) regarding the impact of RT on breast cancer patients over the last 10 years, and differentiate between phases of medical treatment. Furthermore, the review will focus on cancer and treatmentassociated impairments such as reduced muscle strength, secondary lymphedema, body weight and composition changes, reduced bone mineral density, fatigue, and a poorer quality of life (QoL). The literature search was conducted 
in PubMed early in March 2020 and articles were screened for RCTs examining the effects of RT only on breast cancer patients in the period 2010-2020. Additionally, the reference lists of original articles and the reviews of related articles were checked for further eligible studies.

\section{Prehabilitation}

In recent years, evidence has accumulated to suggest that physical fitness prior to breast cancer surgery influences the process of physical recovery [3]. A meta-analysis revealed the potential effects on length of hospital stay (although it was not cancer-specific) [4]. Surprisingly, not many RCTs have been published about prehabilitation of breast cancer patients, and none focused on RT [5]. Most studies discuss combinations of education, aerobic exercise, and mobility exercises $[6,7]$. Strength exercises are positively associated with muscle strength, providing a satisfactory rationale to including RT as a preoperative exercise method. However, to our knowledge, there are no published reports of RCTs only investigating the effects of RT prior to surgery.

\section{Acute Therapy}

Acute treatment options for breast cancer range from surgery, to chemotherapy, hormonal therapy, targeted therapy, immunotherapy, and radiation therapy, often in combination. While there has been a call for targeted studies to determine the correct type, timing, intensity, and duration of exercise [8-10], so as to formulate exercise guidelines for breast cancer patients during acute treatment [11], we only found 4 RCTs and 3 studies with active control groups (Table 1).

\section{Muscle Strength}

RT was found to be safe and effective at improving upper- and lower-body muscular strength in women with breast cancer undergoing acute therapy [12]. The RCTs in the past 10 years have shown beneficial effects on strength parameters during acute therapy.

Ammitzbøll et al. [13] documented increased muscle strength in patients undergoing chemotherapy, by utilizing a progressive, whole-body training protocol that was split into a supervised phase and a longer-term self-administered phase. It is worth mentioning that posttesting was conducted 50 weeks after baseline.

Other mixes of supervised and unsupervised protocols reported positive effects on muscular strength too. Kilbreath et al. [14] trained patients after surgery in a mixed setting comprising a supervised session and a home-based session.
Unsurprisingly, high-intensity training protocols also led to significant strength gains. One study focusing on only 1 machine (the leg-press) and using intensities as high as $85-90 \%$ of the h1RM ([hypothetical 1-repetition maximum], extrapolated hypothetical resistance that the patient is able to overcome once), documented significant strength gains in the lower limbs $[15,16]$. Of this collective, $92 \%$ were designated to undergo chemotherapy, $85 \%$ to have radiation therapy, and $78 \%$ to have hormone therapy, but it is unclear how many patients were actually under treatment during the intervention.

Wiskemann et al. [17] discussed progressive wholebody training and documented significant increases in muscle strength for patients undergoing radiation therapy. A similar approach was documented by Schmidt et al. [18] for patients undergoing chemotherapy, with significant strength gains being reported. Mijwel et al. [19] compared an RT group to an aerobic exercise group, and both collectives also did the same high-intensity interval training (HIIT). They reported significant strength gains for the upper body in the RT group.

\section{BMI/Body Composition}

Neither a high-intensity 12 -week program $[15,16]$ nor a 16-week combination program of RT and HIIT [19] found significant changes in BMI or body composition, even though significant increases in strength were reported for the intervention group with both approaches. Mijwel et al. [19] point out that RT was as efficient as aerobic exercise in maintaining body composition during treatment.

\section{Secondary Lymphedema}

RT has been found to be safe for women surgically treated for breast cancer and does not worsen breast cancer-related lymphedema (BCRL) symptoms [11]. A recent study [13] found no evidence of a preventive effect of RT on BCRL development and no relevant intergroup differences for interlimb volume and mass. Another study [14] using an 8-week RT intervention 4-6 weeks after surgery could not find significant differences between the intervention and control group immediately after the intervention or at the 6-month follow-up. The findings support the safety of RT for patients suffering from BCRL, in accordance with the exercise guidelines [8], suggesting moderate intensities and starting exercise programs in a supervised setting.

\section{Cancer-Related Fatigue}

The exercise guidelines published by Campbell et al. [8] added RT as an effective method for treating cancerrelated fatigue (CRF). A recent study comparing progressive RT and a relaxation group $[17,20]$ during radiation therapy found significant between-group differences for 
Table 1. Resistance exercise in breast cancer patients - RCTs

\begin{tabular}{|c|c|c|c|c|}
\hline $\begin{array}{l}\text { First author } \\
\text { [ref.], year }\end{array}$ & $\begin{array}{l}\text { Supervised vs. unsupervised; } \\
\text { machines vs. free weights }\end{array}$ & Duration & Intensity & Outcome vs. control \\
\hline \multicolumn{5}{|l|}{$\downarrow$ Acute treatment $\downarrow$} \\
\hline $\begin{array}{l}\text { Ammitzbøll } \\
{[13], 2019}\end{array}$ & $\begin{array}{l}1^{\text {st }} 20 \text { weeks, supervised group training + } \\
\text { unsupervised free weights and resistance } \\
\text { bands } \\
2^{\text {nd }} 30 \text { weeks unsupervised }\end{array}$ & $\begin{array}{l}50 \text { weeks, } \\
3 \times / \text { week }\end{array}$ & $\begin{array}{l}\text { Whole body, progressive, } 10-20 \text { reps, } \\
3 \text { sets }\end{array}$ & $\begin{array}{l}\uparrow \text { Muscle strength } \\
\leftrightarrow \text { BCRL volume } \\
\leftrightarrow \text { BCRL symptoms }\end{array}$ \\
\hline $\begin{array}{l}\text { Cešeiko } \\
{[15], 2019}\end{array}$ & Supervised, leg-press machine & $\begin{array}{l}12 \text { weeks, } \\
2 \times / \text { week for } 20 \mathrm{~min}\end{array}$ & $\begin{array}{l}\text { Leg-press, progressive, } 4 \text { reps, } 4 \text { sets } \\
\text { at } 85-90 \% \text { h1RM }\end{array}$ & $\begin{array}{l}\uparrow \text { Muscle strength } \\
\leftrightarrow \text { BMI } \\
\uparrow \text { QoL (EORTC-QLQ C30, BR23) }\end{array}$ \\
\hline $\begin{array}{l}\text { Kilbreath } \\
{[14], 2012}\end{array}$ & $\begin{array}{l}\text { Supervised }+ \text { unsupervised } \\
\text { Free weights, resistance bands }\end{array}$ & $\begin{array}{l}8 \text { weeks, } \\
1 \times / \text { week + home program, } \\
24 \text {-week follow-up }\end{array}$ & $\begin{array}{l}\text { Shoulder muscles, exercises } \\
\text { unspecified progressive, } 8-15 \text { reps }\end{array}$ & $\begin{array}{l}\uparrow \text { Muscle strength } \\
\leftrightarrow \text { BCRL } \\
\uparrow \text { BCRL symptoms }\end{array}$ \\
\hline $\begin{array}{l}\text { Mijwel } \\
{[19], 2018}\end{array}$ & $\begin{array}{l}\text { RE + HIIT: supervised, machines } \\
\text { AE + HIIT: supervised }\end{array}$ & $\begin{array}{l}\text { RE/AE: } 16 \text { weeks during } \\
\text { chemotherapy, } \\
2 \times / \text { week }\end{array}$ & $\begin{array}{l}\text { RE: } 8-12 \text { reps, } 2-3 \text { sets } 80 \% \text { h1RM } \\
3 \times 3 \text { min HIIT } \\
\text { AE: } 20 \text { min steady state, } 13-15 \\
\text { BORG Scale } \\
3 \times 3 \text { min HIIT }\end{array}$ & $\begin{array}{l}\text { RE+HIIT vs. AE+HIIT: } \\
\uparrow \text { Muscle strength } \\
\leftrightarrow \text { BMI } \\
\uparrow \text { Pressure-pain threshold }\end{array}$ \\
\hline $\begin{array}{l}\text { Schmidt } \\
{[21], 2015}\end{array}$ & $\begin{array}{l}\text { RE: group training, supervised } \\
\text { RELX: group training, supervised }\end{array}$ & $\begin{array}{l}12 \text { weeks, } \\
2 \times / \text { week during } \\
\text { chemotherapy }\end{array}$ & $\begin{array}{l}\text { Whole body, progressive, } 3 \text { sets/ } \\
12 \text { rep max } \\
\text { Progressive muscle relaxation }\end{array}$ & $\begin{array}{l}\text { RE vs. RELX } \\
\uparrow \text { CRF } \\
\leftrightarrow \text { QoL }\end{array}$ \\
\hline $\begin{array}{l}\text { Schmidt } \\
{[18], 2015}\end{array}$ & Supervised, machines & $\begin{array}{l}12 \text { weeks, } \\
2 \times / \text { week during } \\
\text { chemotherapy }\end{array}$ & $\begin{array}{l}\text { Whole body, progressive, } 20 \text { reps, } \\
1 \text { set at } 50 \% \text { h1RM }\end{array}$ & $\begin{array}{l}\uparrow \text { Muscle strength } \\
\leftrightarrow \mathrm{CRF} \\
\uparrow \mathrm{QoL}\end{array}$ \\
\hline $\begin{array}{l}\text { Steindorf } \\
{[20], 2014 /} \\
\text { Wiskemann } \\
{[17], 2017}\end{array}$ & $\begin{array}{l}\text { RE: machines unsupervised } \\
\text { RELX: group training, supervised }\end{array}$ & $\begin{array}{l}12 \text { weeks, } \\
2 \times / \text { week }\end{array}$ & $\begin{array}{l}\text { RE: whole body, progressive, } \\
3 \text { sets/12 rep max } \\
\text { RELX: PMR }\end{array}$ & $\begin{array}{l}\text { RE vs. RELX } \\
\uparrow \text { Muscle strength } \\
\leftrightarrow \text { CRF }\end{array}$ \\
\hline \multicolumn{5}{|l|}{$\downarrow$ Survivors $\downarrow$} \\
\hline $\begin{array}{l}\text { Schmitz } \\
{[28], 2010 /} \\
\text { Brown } \\
{[27], 2012}\end{array}$ & $\begin{array}{l}1^{\text {st }} 3 \text { months supervised; } \\
2^{\text {nd }} 9 \text { months unsupervised } \\
\text { Machines and free weights }\end{array}$ & $\begin{array}{l}1 \text { year, } \\
2 \times / \text { week for } 90 \mathrm{~min}\end{array}$ & $\begin{array}{l}\text { Whole body, progressive, } 10 \text { reps, } \\
3 \text { sets }\end{array}$ & $\begin{array}{l}\uparrow \text { Muscle strength } \\
\uparrow \text { BCRL onset } \\
\leftrightarrow \text { BMI } \\
\leftrightarrow \text { Lean mass }\end{array}$ \\
\hline $\begin{array}{l}\text { Winters-Stone } \\
{[35], 2014}\end{array}$ & As above & As above & As above & $\leftrightarrow \mathrm{BMD}$ \\
\hline $\begin{array}{l}\text { Hagstrom } \\
{[24], 2019}\end{array}$ & $\begin{array}{l}\text { Supervised; } 1^{\text {st }} 8 \text { weeks machines, } \\
2^{\text {nd }} 8 \text { weeks free weights }\end{array}$ & $\begin{array}{l}16 \text { weeks, } \\
3 \times / \text { week for } 60 \mathrm{~min}\end{array}$ & $\begin{array}{l}\text { Whole body, progressive, } 8-10 \text { reps, } \\
3 \text { sets }\end{array}$ & $\uparrow$ Muscle strength \\
\hline $\begin{array}{l}\text { Hagstrom } \\
{[23], 2016 /} \\
\text { Hagstrom } \\
{[41], 2016}\end{array}$ & As above & As above & As above & $\begin{array}{l}\uparrow \text { Muscle strength } \\
\leftrightarrow \text { BMI } \\
\uparrow \text { QoL (FACT-G) } \\
\uparrow \text { Fatigue (FACIT) }\end{array}$ \\
\hline $\begin{array}{l}\text { Dos Santos } \\
{[26], 2019}\end{array}$ & $\begin{array}{l}\text { Supervised, } \\
\text { Machines and free weights }\end{array}$ & $\begin{array}{l}8 \text { weeks, } \\
1 \times / \text { week }\end{array}$ & $\begin{array}{l}\text { Whole body, progressive, } 8-12 \text { reps, } \\
3 \text { sets }\end{array}$ & $\begin{array}{l}\uparrow \text { Muscle strength } \\
\leftrightarrow \text { BMI } \\
\leftrightarrow \text { Lean mass }\end{array}$ \\
\hline $\begin{array}{l}\text { Jeffs } \\
{[31], 2013}\end{array}$ & $\begin{array}{l}\text { Unsupervised } \\
\text { Gravity resistive exercises }\end{array}$ & $\begin{array}{l}6 \text { months, } \\
7 \times / \text { week for } 10-15 \mathrm{~min}\end{array}$ & Upper body, no intensities reported & $\begin{array}{l}\leftrightarrow \text { BCRL volume } \\
\leftrightarrow \text { QoL (LYMQOL) } \\
\leftrightarrow \text { BMI }\end{array}$ \\
\hline $\begin{array}{l}\text { Waltman } \\
{[36], 2010}\end{array}$ & $\begin{array}{l}\text { Unsupervised, } \\
1^{\text {st }} 9 \text { months: free weights } \\
2^{\text {nd }} 15 \text { months: machines }\end{array}$ & $\begin{array}{l}24 \text { months, } \\
2 \times / \text { week }\end{array}$ & $\begin{array}{l}\text { Whole body, progressive } \\
8-12 \text { reps, } 2 \text { sets }\end{array}$ & $\leftrightarrow \mathrm{BMD}$ \\
\hline $\begin{array}{l}\text { Santagnello } \\
{[37], 2020}\end{array}$ & Supervised, machines & $\begin{array}{l}12 \text { weeks, } \\
3 \times / \text { week }\end{array}$ & $\begin{array}{l}\text { Lower body, progressive, } 8-12 \text { reps, } \\
3 \text { sets }\end{array}$ & $\begin{array}{l}\uparrow \text { Muscle strength } \\
\uparrow \text { Lean mass } \\
\uparrow \text { Fatigue }\end{array}$ \\
\hline $\begin{array}{l}\text { Buchan } \\
{[34], 2016}\end{array}$ & $\begin{array}{l}\text { RE: } 1 / 3 \text { supervised and } 2 / 3 \text { unsupervised, } \\
\text { machines and free weights } \\
\text { AE: } 1 / 3 \text { supervised and } 2 / 3 \text { unsupervised }\end{array}$ & $\begin{array}{l}\text { RE/AE: } 12 \text { weeks }+ \\
\text { 12-week follow-up, } \\
3 \times / \text { week for } 50 \mathrm{~min}\end{array}$ & $\begin{array}{l}\text { RE: Whole body, progressive, } \\
8-12 \text { reps, } 2 \text { sets } \\
\text { AE: intensities according a } \\
\text { MET-level of } 5\end{array}$ & $\begin{array}{l}\text { RE vs. AE: } \\
\uparrow \text { Muscle strength } \\
\leftrightarrow \text { BCRL } \\
\leftrightarrow \text { Lean mass } \\
\leftrightarrow \text { QoL (FACT-B) }\end{array}$ \\
\hline
\end{tabular}


Table 1 (continued)

\begin{tabular}{|c|c|c|c|c|}
\hline $\begin{array}{l}\text { First author } \\
\text { [ref.], year }\end{array}$ & $\begin{array}{l}\text { Supervised vs. unsupervised; } \\
\text { machines vs. free weights }\end{array}$ & Duration & Intensity & Outcome vs. control \\
\hline $\begin{array}{l}\text { Musanti } \\
{[25], 2012}\end{array}$ & $\begin{array}{l}\text { Unsupervised } \\
\text { RE: free weights (resistance bands) } \\
\text { AE: walking } \\
\text { REAE: combination of both }\end{array}$ & $\begin{array}{l}12 \text { weeks, } \\
\text { RE: } 3 \times / \text { week } \\
\text { AE: } 3 \times / \text { week for } 15-30 \mathrm{~min} \\
\text { REAE: RE } 2 \times / \text { week }+ \text { AE } \\
4-5 \times / \text { week }\end{array}$ & $\begin{array}{l}\text { RE: whole body, progressive, } \\
10-12 \text { reps, } 1 \text { set } \\
\text { AE: } 40-65 \% \text { (up to } 85 \% \text { ) of HRmax } \\
\text { Combination of both, } \\
\text { intensities as above }\end{array}$ & $\begin{array}{l}\text { RE and REAE vs. AE and controls: } \\
\uparrow \text { Muscle strength }\end{array}$ \\
\hline $\begin{array}{l}\text { Cormie } \\
{[29], 2013}\end{array}$ & $\begin{array}{l}\text { Supervised; machines and free weights } \\
\text { RE intense } \\
\text { RE moderate }\end{array}$ & $\begin{array}{l}12 \text { weeks } \\
2 \times / \text { week for } 60 \mathrm{~min}\end{array}$ & $\begin{array}{l}\text { Whole body, progressive, } \\
\text { RE intense: } 6-10 \text { reps with } \\
75-85 \% \text { of } 1 \mathrm{RM}, 1-4 \text { sets } \\
\text { RE moderate: } 15-20 \text { reps with } \\
55-65 \% \text { of } 1 \mathrm{RM}, 1-4 \text { sets }\end{array}$ & $\begin{array}{l}\text { RE HIGH and MOD vs. controls: } \\
\leftrightarrow \text { BCRL volume } \\
\leftrightarrow \text { BCRL symptoms } \\
\uparrow \text { Muscle strength }\end{array}$ \\
\hline
\end{tabular}

Overview of strength exercise RCT studies in acute breast cancer therapy and in breast cancer survivors over the last 10 years. AE, aerobic exercise; BCRL, breast cancer-related lymphedema; BMD, bone mineral density; BMI, body mass index; CRF, cancer-related fatigue; HIIT, high-intensity interval training; HRmax, maximum heart rate; MET, metabolic equivalent of task; PMR, progressive muscle relaxation; RE, resistance exercise; REAE, resistance + aerobic exercise; RELX, relaxation training; reps, repetitions; QoL, quality of life.

self-reported CRF using the Fatigue Assessment Questionnaire (FAQ), especially concerning physical fatigue, but both interventions led to improvements in total and affective scores. Muscular fatigue worsened from baseline to postintervention in the relaxation group, but there was no deterioration in the RT group. Using comparable training designs with collectives undergoing chemotherapy, a study on lower-intensity RT [18] did not associate RT with improvements in CRF; a study on higher-intensity RT [21] found a favorable tendency for RT compared to relaxation training.

\section{Quality of Life}

QoL has been reported to be affected positively by RT during adjuvant therapy [22], and Campbell et al. [8] recommend $2-3$ sets of $8-15$ repetitions at $60-75 \%$ of the $1 \mathrm{RM}$ for cancer patients. In the recommended supervised setting, but using higher intensities, Cešeiko et al. [15] reported positive effects for several scales in the EORTC QLQ C30 and BR23 questionnaires. One study on using low intensities during chemotherapy [18] found significant improvements in QoL, while a comparable design utilizing moderate intensities suggests slight improvements for role and social function, and a maintenance of global QoL measures. During radiotherapy, another study [20] reported significant increases in global QoL, again using a comparable training protocol.

\section{Breast Cancer Survivors}

\section{Muscle Strength}

Strength training is widely known to be beneficial in breast cancer survivors especially due to its effects on muscle function. Clinical trials in the last 10 years substantiated this assumption with conclusive findings of improvements of muscle strength following strength exercise interventions (Table 1) [23-29, 37].

Three RCTs [23, 24, 26] showed improved muscle strength due to machine-based and free-weight strength exercise programs of 16 and 8 weeks, respectively. The latter exercise program [26] was conducted as a 1:1 patient-trainer supervision, suggesting the ideal exercise support, whereas Hagstrom et al. [23, 24] used a smallgroup setting. An equivalent strength training protocol, with machine-based exercises of the lower body only, led to improved muscle strength after 12 weeks [37]. The Physical Activity and Lymphedema (PAL) trial showed that an initial 3 months of supervised strength exercise, followed by 9 months unsupervised, improved muscle strength by using comparable exercise protocols.

In comparison to aerobic exercise, strength exercise seems to be crucial for muscle strength improvements. Twelve weeks of mixed home-based and supervised RT showed superior muscle strength improvements in the upper body than 12 weeks of mixed home-based and supervised aerobic exercise [34]. The combination of aerobic exercise and RT does not seem to be more effective than RT only. The trial of Musanti [25] reported similar significant increases of muscle strength in breast cancer survivors who performed strength training or combined strength and aerobic training compared to aerobic- or mobility-exercised groups.

The study of Cormie et al. [29] showed that moderate RT was sufficient to improve muscle strength in the same way as intense strength training, with significant improvements in both groups compared to a control group. It should be mentioned that their study did not explicitly list completed treatment as an inclusion criterion, but the mean time since cancer diagnosis of the study population was given as approximately 6 years. 


\section{BMI/Body Composition}

Both short- and long-term exercise durations did not change the BMI in a significant manner using RT protocols that lasted 1 year [25], nor did daily gravity-resistive exercise for 6 months [28]. Likewise, short- and mid-term strength exercise led to no changes after 16 weeks of strength exercise [23] and only small increases in BMI following 8 weeks of personal training [28]. Despite improved muscle strength, only 1 of 4 studies showed an impact of RT on lean body mass [34].

\section{Secondary Lymphedema}

Current reviews conclude that resistance exercise is safe and does not exacerbate BCRL $[29,30]$. The strength studies of the last 10 years are in line with these conclusions. The PAL trial [25] reported a significantly reduced number of women experiencing a BCRL incident or onset in the weightlifting group after 1 year of exercise. However, 2 studies with shorter exercise duration reported no effects of exercise on the volume $[29,31]$, number of symptoms, or severity of BCRL [29]. Comparison of 12 weeks of aerobic and strength exercise did not reveal differences in BCRL domains [34].

\section{Bone Health}

Two trials investigated the effects of RT only on bone mineral density (BMD). Neither study found any significant changes in BMD in breast cancer survivors after 1 or 2 years of strength exercise $[35,36]$. However, small and nonsignificant trends in both studies suggested a potential osteogenic effect of RT in postmenopausal women.

\section{Cancer-Related Fatigue}

Two recent studies on breast cancer survivors evaluated the effects of progressive RT on CRF. Subjectively perceived fatigue improved in survivors who undertook RT compared to the control group [37, 41].

\section{Quality of Life}

Whereas the study of Jeffs and Wiseman [31] did not show increases of lymphedema-related QoL after 6 months of daily gravity-resistive exercises, the trial of Hagstrom et al. [41] improved breast cancer survivors' QoL (according to the functional assessment of cancer therapy-general [FACT-G] scale) following whole-body strength training over the same period. When the 12week strength and aerobic exercise were compared, no differences were observed [34].

\section{Discussion}

In the current national guidelines, $\mathrm{RT}$ is an established supportive treatment during adjuvant therapy and for long-term survivors, and it is recommended for use dur- ing chemotherapy, antihormone treatment, and in the presence of BCRL in the German Breast Cancer Guidelines [42]. It is considered a flexible, proven, and powerful tool to treat muscle loss, reduced strength, CRF, lymphedema, and several aspects of QoL $[8,38,39]$. Furthermore, RT was found to be safe even utilizing high loads during adjuvant treatment [12]. However, current recommendations for cancer patients are vague. There is not enough evidence to provide guidelines for individualized, standardized, and targeted interventions that take into account therapy stage and other factors that influence the goals of interventions.

The listed studies underline the efficacy of RT for breast cancer patients during treatment and aftercare with regard to muscle strength. Supervised and unsupervised settings proved to be efficient as well as a broad range of intensity and length of periods of exercise. The studies of the past decade were generally useful in showing the positive impact of several factors on patients' QoL. RT as a treatment for CRF is now implemented in the exercise guidelines for cancer patients [8] despite the scarcity of pure RT studies in the past 10 years; effect sizes apparently depend on training intensity $[18,20]$. The findings on RT and the development and symptomatology of lymphedemas vary, conclusively only suggesting the feasibility and safety but no distinct benefits of RT in patients with lymphedemas. Considering changes in BMI, aerobic exercise seems to be more beneficial for cancer patients, although the possible impact of RT on body composition is not reflected in the cited studies.

However, these studies cannot do justice to the complexity of breast cancer treatment and the diversity of RT. The lack of studies examining RT in prehabilitation, and the small number of RT-only protocols in adjuvant therapy was especially surprising, as surgery is associated with a loss of muscular strength and a reduced range of motion.

Comparability of the studies included was extremely limited due to different testing techniques, different questionnaires, different (and often not clearly outlined) training protocols, and a lack of information about the timing of inclusion and testing.

RT can be carried out indoors and outdoors in supervised, group, or home-based settings that utilize machines, free weights, and other equipment. Lund et al. [40] documented that supervision and support is crucial for adherence in untrained and overweight patients. RT can be considered a highly flexible therapeutic tool.

The measurability of RT exercises through resistance, range of motion, repetitions, and sets predestines this modality for systematic study designs. Simple tools for training control need to be established to meet target intensity and to create safe and efficient guidelines. 
Regular physical activity is associated with reduced breast cancer-specific mortality and recurrence [43]. New data suggest that RT may lower chronic inflammation, thereby highlighting its potential to influence one major driver of cancer progression or recurrence [44]. Such perspectives emphasize the importance of a higher output of studies in the field of RT for breast cancer, studies that focus on the promising effects of RT on inflammation, bone health, and long-term survival. Currently, there is too little information comparing RT to other interventions to be able to determine which patients can benefit most from each type of exercise intervention.

\section{Conclusion}

Breast cancer patients are a very heterogeneous collective and the goals at every stage of therapy vary. The RCT of the last decade emphasised the beneficial impact of RT on muscle strength, CRF, QoL and possibly lymphedema in breast cancer patients during medical treatment and in survivorship with differing evidence levels. Therefore, a need for individualized guidelines for breast cancer patients exists, that will take into account therapy stage, medication, fitness, and other parameters. The studies of the past decade have found evidence for RT only in the supportive care of breast cancer, but there is room for specifically targeted study designs that will facilitate the compilation of individualized recommendations.

\section{Conflict of Interest Statement}

The authors have no conflicts of interest to declare.

\section{Funding Sources}

This research received no specific grant from any funding agency in the public, commercial, or not-for-profit sectors.

\section{Author Contributions}

L.G., F.T.B., and T.N.: study conceptualization, methodology, and writing the paper. L.G. and T.N.: investigation.

\section{References}

1 Wild CP, Weiderpass E, Stewart BW, editors. (2020): World Cancer Report: Cancer Research for Cancer Prevention. Lyon, France: International Agency for Research on Cancer. Available from: http://publications.iarc. fr/586. Licence: CC BY-NC-ND3.0 IGO.

2 Lee J, Lee MG. Effects of exercise intervention on breast cancer patients during adjuvant therapy: A systematic review and meta-analysis of randomized controlled trials. Cancer Nurs. 2020 Mar/Apr;43(2):115-25.

3 Nilsson H, Angerås U, Bock D, Börjesson M, Onerup A, Fagevik Olsen M, et al. Is preoperative physical activity related to post-surgery recovery? A cohort study of patients with breast cancer. BMJ Open. 2016 Jan; 6(1):e007997.

4 Santa Mina D, Clarke H, Ritvo P, Leung YW, Matthew AG, Katz J, et al. Effect of total-body prehabilitation on postoperative outcomes: a systematic review and meta-analysis. Physiotherapy. 2014 Sep;100(3):196-207.

5 Yang A, Sokolof J, Gulati A. The effect of preoperative exercise on upper extremity recovery following breast cancer surgery: a systematic review. Int J Rehabil Res. 2018 Sep;41(3): 189-96.

6 Lokapavani Y, Ragava Krishna S, Madhavi K. Influence of pre-operative physical therapy education and exercise on post-operative shoulder range of motion and functional activities in subjects with modified radical mastectomy. In: int. J Physiother. 2014;1(4):1707.

7 Baima J, Reynolds SG, Edmiston K, Larkin A, Ward BM, O'Connor A. Teaching of independent exercises for Prehabilitation in breast cancer. J Cancer Educ. 2017 Jun;32(2):252-6.
8 Campbell KL, Winters-Stone KM, Wiskemann J, May AM, Schwartz AL, Courneya KS, et al. Exercise Guidelines for Cancer Survivors: Consensus Statement from International Multidisciplinary Roundtable. Med Sci Sports Exerc. 2019 Nov;51(11):2375-90.

9 Dieli-Conwright CM, Orozco BZ. Exercise after breast cancer treatment: current perspectives. Breast Cancer (Dove Med Press). 2015 Oct; 7:353-62.

10 Baumann FT, Bloch W, Weissen A, Brockhaus M, Beulertz J, Zimmer P, et al. Physical Activity in Breast Cancer Patients during Medical Treatment and in the Aftercare - a Review. Breast Care (Basel). 2013 Oct;8(5):330-4.

11 Courneya KS, McKenzie DC, Mackey JR, Gelmon K, Friedenreich CM, Yasui Y, et al. Subgroup effects in a randomised trial of different types and doses of exercise during breast cancer chemotherapy. Br J Cancer. 2014 Oct; 111(9):1718-25.

12 Cheema BS, Kilbreath SL, Fahey PP, Delaney GP, Atlantis E. Safety and efficacy of progressive resistance training in breast cancer: a systematic review and meta-analysis. Breast Cancer Res Treat. 2014 Nov;148(2):249-68.

13 Ammitzbøll G, Johansen C, Lanng C, Andersen EW, Kroman N, Zerahn B, et al. Progressive resistance training to prevent arm lymphedema in the first year after breast cancer surgery: results of a randomized controlled trial. Cancer. 2019 May;125(10):1683-92.

14 Kilbreath SL, Refshauge KM, Beith JM, Ward LC, Lee M, Simpson JM, et al. Upper limb progressive resistance training and stretching exercises following surgery for early breast cancer: a randomized controlled trial. Breast Cancer Res Treat. 2012 Jun;133(2):667-76.
15 Cešeiko R, Eglītis J, Srebnijs A, Timofejevs M, Purmalis E, Erts R, et al. The impact of maximal strength training on quality of life among women with breast cancer undergoing treatment. Exp Oncol. 2019 Jun;41(2):166-72.

16 Cešeiko R, Thomsen SN, Tomsone S, Eglitis J, Vētra A, Srebnijs A, et al. Heavy Resistance Training in Breast Cancer Patients Undergoing Adjuvant Therapy. Med Sci Sports Exerc. 2020 Jun;52(6):1239-47.

17 Wiskemann J, Schmidt ME, Klassen O, Debus J, Ulrich CM, Potthoff K, et al. Effects of 12week resistance training during radiotherapy in breast cancer patients. Scand J Med Sci Sports. 2017 Nov;27(11):1500-10.

18 Schmidt T, Weisser B, Dürkop J, Jonat W, Van Mackelenbergh M, Röcken C, et al. Comparing Endurance and Resistance Training with Standard Care during Chemotherapy for Patients with Primary Breast Cancer. Anticancer Res. 2015 Oct;35(10):5623-9.

19 Mijwel S, Backman M, Bolam KA, Olofsson E, Norrbom J, Bergh J, et al. Highly favorable physiological responses to concurrent resistance and high-intensity interval training during chemotherapy: the OptiTrain breast cancer trial. Breast Cancer Res Treat. 2018 May;169(1):93-103.

20 Steindorf K, Schmidt ME, Klassen O, Ulrich CM, Oelmann J, Habermann N, et al. Randomized, controlled trial of resistance training in breast cancer patients receiving adjuvant radiotherapy: results on cancer-related fatigue and quality of life. Ann Oncol. 2014 Nov;25(11):2237-43. 
21 Schmidt ME, Wiskemann J, Armbrust P, Schneeweiss A, Ulrich CM, Steindorf K. Effects of resistance exercise on fatigue and quality of life in breast cancer patients undergoing adjuvant chemotherapy: A randomized controlled trial. Int J Cancer. 2015 Jul;137(2): 471-80.

22 Carayol M, Bernard P, Boiché J, Riou F, Mercier B, Cousson-Gélie F, et al. Psychological effect of exercise in women with breast cancer receiving adjuvant therapy: what is the optimal dose needed? Ann Oncol. 2013 Feb;24(2): 291-300.

23 Hagstrom AD, Marshall PW, Lonsdale C, Papalia S, Cheema BS, Toben C, et al. The effect of resistance training on markers of immune function and inflammation in previously sedentary women recovering from breast cancer: a randomized controlled trial. Breast Cancer Res Treat. 2016 Feb;155(3):471-82.

24 Hagstrom AD, Shorter KA, Marshall PW. Changes in Unilateral Upper Limb Muscular Strength and Electromyographic Activity After a 16-Week Strength Training Intervention in Survivors of Breast Cancer. J Strength Cond Res. 2019 Jan;33(1):225-33.

25 Musanti R. A study of exercise modality and physical self-esteem in breast cancer survivors. Med Sci Sports Exerc. 2012 Feb;44(2): 352-61.

26 Dos Santos WD, Vieira A, de Lira CA, Mota JF, Gentil P, de Freitas Junior R, et al. Once a Week Resistance Training Improves Muscular Strength in Breast Cancer Survivors: A Randomized Controlled Trial. Integr Cancer Ther. 2019 Jan-Dec; 18:1534735419879748.

27 Brown JC, Troxel AB, Schmitz KH. Safety of weightlifting among women with or at risk for breast cancer-related lymphedema: musculoskeletal injuries and health care use in a weightlifting rehabilitation trial. Oncologist. 2012;17(8):1120-8.

28 Schmitz KH, Ahmed RL, Troxel AB, Cheville A, Lewis-Grant L, Smith R, et al. Weight lifting for women at risk for breast cancer-related lymphedema: a randomized trial. JAMA. 2010 Dec;304(24):2699-705
29 Cormie P, Pumpa K, Galvão DA, Turner E, Spry N, Saunders C, et al. Is it safe and efficacious for women with lymphedema secondary to breast cancer to lift heavy weights during exercise: a randomised controlled trial. J Cancer Surviv. 2013 Sep;7(3):413-24.

30 Wiskemann J, Clauss D, Tjaden C, Hackert T, Schneider L, Ulrich CM, et al. Progressive Resistance Training to Impact Physical Fitness and Body Weight in Pancreatic Cancer Patients: A Randomized Controlled Trial. Pancreas. 2019 Feb;48(2):257-66.

31 Jeffs E, Wiseman T. Randomised controlled trial to determine the benefit of daily homebased exercise in addition to self-care in the management of breast cancer-related lymphoedema: a feasibility study. Support Care Cancer. 2013 Apr;21(4):1013-23.

32 Wanchai A, Armer JM. Effect of weight-lifting or resistance exercise on breast cancerrelated lymphedema: A systematic review. Int J Nurs Sci. 2018;6(1):92-8.

33 Keilani M, Hasenoehrl T, Neubauer M, Crevenna R. Resistance exercise and secondary lymphedema in breast cancer survivors-a systematic review. Support Care Cancer. 2016 Apr;24(4):1907-16.

34 Buchan J, Janda M, Box R, Schmitz K, Hayes $S$. A randomized trial of the effect of exercise mode on breast-cancer related lymphedema. Med Sci Sports Exerc. 2016 Oct;48(10):186674.

35 Winters-Stone KM, Laudermilk M, Woo K, Brown JC, Schmitz KH. Influence of weight training on skeletal health of breast cancer survivors with or at risk for breast cancer-related lymphedema. J Cancer Surviv. 2014 Jun; 8(2):260-8.

36 Waltman NL, Twiss JJ, Ott CD, Gross GJ, Lindsey AM, Moore TE, et al. The effect of weight training on bone mineral density and bone turnover in postmenopausal breast cancer survivors with bone loss: a 24-month randomized controlled trial. Osteoporos Int. 2010 Aug;21(8):1361-9.

37 Santagnello SB, Martins FM, De Oliveira Junior GN, de Freitas Rodrigues de Sousa J,
Normelini RS, Murta EF, et al. Improvements in muscle strength, power, and size and selfreported fatigue as mediators of the effect of resistance exercise on physical performance breast cancer survivor women: a randomized controlled trial. Support Care Cancer. 2020 Dec;28(12):6075-84.

38 Baena-Cañada JM, Estalella-Mendoza S, González-Guerrero M, Expósito-Álvarez I, Rosado-Varela P, Benítez-Rodríguez E. [Influence of clinical and biographical factors on the quality of life of women with breast cancer receiving adjuvant chemotherapy]. Rev Calid Asist. 2011 Sep-Oct;26(5):299-305.

39 Stan D, Loprinzi CL, Ruddy KJ. Breast cancer survivorship issues [ix.]. Hematol Oncol Clin North Am. 2013 Aug;27(4):805-27.

40 Lund LW, Ammitzbøll G, Hansen DG, Andersen EA, Dalton SO. Adherence to a long-term progressive resistance training program, combining supervised and home-based exercise for breast cancer patients during adjuvant treatment. Acta Oncol. 2019 May;58(5):650-7.

41 Hagstrom AD, Marshall PW, Lonsdale C, Cheema BS, Fiatarone Singh MA, Green S. Resistance training improves fatigue and quality of life in previously sedentary breast cancer survivors: a randomised controlled trial. Eur J Cancer Care (Engl). 2016 Sep;25(5):784-94.

42 Onkologie L. (Deutsche Krebsgesellschaft, Deutsche Krebshilfe, AWMF): S3-Leitlinie Früherkennung, Diagnose, Therapie und Nachsorge des Mammakarzinoms, Version 4.2, 2020 AWMF Registernummer: 032045OL, http://www.leitlinienprogrammonkologie.de/leitlinien/mammakarzinom/ (access date: 23.04.2020).

43 Ibrahim EM, Al-Homaidh A. Physical activity and survival after breast cancer diagnosis: meta-analysis of published studies. Med Oncol. 2011 Sep;28(3):753-65.

44 Winters-Stone KM, Wood LJ, Stoyles S, Dieckmann NF. The Effects of Resistance Exercise on Biomarkers of Breast Cancer Prognosis: A Pooled Analysis of Three Randomized Trials. Cancer Epidemiol Biomarkers Prev. 2018 Feb;27(2):146-53. 